\title{
OPTIMAL STRUCTURAL DESIGN VIA OPTIMALITY CRITERIA AS A NONSMOOTH MECHANICS PROBLEM
}

\author{
M. Ap. Tzaferopoulos $\nmid$ and G. E. Stavroulakis $\Varangle \S$ \\ †Cambridge University, Engineering Department, Trumpington Street, Cambridge CB2 1PZ, U.K. \\ tAlexander von Humboldt Research Fellow, Lehr- und Forschungesbebiet für Mechanik, \\ RWTH Aachen Templergraben 64, D-52062 Aachen, Germany
}

\author{
(Received 18 December 1993)
}

\begin{abstract}
In the theory of plastic structural design via optimality criteria (due to $\mathrm{W}$. Prager), the optimal design problem is transformed to a nonlinear elastic structural analysis problem with appropriate stress-strain laws, which generally include complete vertical branches. In this context, the concept of structural universe (in the sense of G. Rozvany) permits the treatment of complicated optimal layout problems. Recent progress in the field of nonsmooth mechanics makes the solution of structural analysis problems with this kind of "complete" law possible. Elements from the two fields are combined in this paper for the solution of optimal design and layout problems for structures. The optimal layout of plane trusses with various specific cost functions is studied here as a representative problem. The use of convex, continuous and piecewise linear specific cost functions for the structural members leads to problems of linear variational inequalities or equivalently piecewise linear, convex but nonsmooth optimization problems, which are solved by means of an iterative algorithm based on sequential linear programming techniques. Numerical examples illustrate the theory and its applicability to practical engineering structures. Following a parametric investigation of an optimal bridge design, certain aspects of the optimal truss layout problem are discussed, which can be extended to other types of structural systems as well.
\end{abstract}

\section{INTRODUCTION}

In this paper the problem of optimal plastic design and layout of structures is studied by means of the optimality criteria technique, which was first introduced by Prager and Shield [1], and the concept of structural universe, which was introduced by Rozvany [2]. We also use certain results from the field of nonsmooth (or inequality) mechanics (a term introduced by Panagiotopoulos, see [3-6]) for the solution of highly nonlinear structural analysis problems, concerning structures with complete stressstrain laws (i.e. laws which have complete vertical branches).

The optimal design problem can be treated by direct application of optimization methods to the parametric structural model. General purpose mathematical programming techniques are used in a black-box style, which makes inefficient use of computational resources and thus restricts the applications to small-scale problems $[7,8]$. Those drawbacks notwithstanding, this approach is employed in most cases today, and in this form it is incorporated in general finite element programs (e.g. in the optimization routines of NASTRAN).

\& On leave from: Institute of Applied Mechanics, Department of Engineering Sciences, Technical University of Crete, GR-73100 Chania, Greece. To whom correspondence should be addressed.
Alternatively, for the solution of the optimal design problem, the optimality criteria [2] approach may be used. This method was initially proposed by Prager [1] for the optimal plastic design problem and was subsequently extended to several elastic and plastic design problems $[2,7-9]$. The optimality criteria are conditions which must be fulfilled at the solution of an optimal design problem $[1,2,10]$. Closed-form and analytic solutions have been obtained for some relatively simple structures only with simple specific cost functions (cf. [2, 11-14] where optimal design problems for trusses, frameworks and plates are presented).

A feature of the latter approach that will be exploited in this paper is the fact that, under certain conditions on the adopted specific cost function (it must be a function of the stress components at each point of the structure), the optimal plastic design problem may be transformed to a nonlinear elastic structural analysis problem. The stress-strain relation (constitutive law) assumed during the analysis of the "associated" fictitious structure is derived by generalized differentiation from the specific cost function in conjunction with the optimality criteria. Those stress-strain laws are monotone and nonmonotone complete relations (i.e. they may include ascending and descending complete vertical branches). Existing general nonlinear structural analysis methods are not able to treat effectively the arising highly nonlinear (nonsmooth) problems, because of the underlying 
smoothness assumptions. Material and boundary laws of this type have recently been studied in the field of nonsmooth mechanics $[3,4,6,15$ 17] and considerable experience has been accumulated in their treatment. Using the tools developed in this context, the method of optimal plastic design can be expanded in the study of practical large-scale structures. Nonsmooth specific cost functions arise frequently in applications and in general they represent the effect of various design concepts of production techniques which can be used for the fabrication of structural members.

The branch of nonsmooth mechanics is concerned with the analysis of mechanical systems and structures whose mechanical behavior is described by inequalities or generally nonsmooth functions $[3,4,6]$. It is an outgrowth of the study of primitive inequality mechanics problems [5] (e.g. the unilateral contact problem and the Coulomb friction problem) and embodies results from the fields of convex and nonconvex analysis, nonsmooth analysis, the theory of variational inequalities and optimization (the reader is referred to Refs $[15,18-20]$ for an extended exposition).

General constitutive laws or boundary conditions which can be written in the form of a monotone or nonmonotone multivalued function, as is the case with most optimality conditions arising in optimal structural design, may be produced from convex and nonconvex, nonsmooth potential or complementary energy expressions. Such superpotentials are similar to the (nonsmooth) specific cost functions of Prager's theory and can be manipulated by means of generalizations of the classical differential operator of smooth analysis: the subdifferential operator of convex analysis is uscd for monotonc laws, whilc for nonmonotone laws the generalized subdifferential in the sense of Clarke, or the quasidifferential operator in the sense of Demyanov is introduced. The variational $[5,6,18]$ and hemivariational $[3,4,6,20]$ inequality problems that arise this way are equivalent to convex and nonconvex optimization problems or generally substationarity problems, under certain assumptions. Thus a sufficiently general theory has been developed for the analysis of structures with nonsmooth mechanical behavior and/or free boundary problems: unilateral contact problems with or without friction effects, plasticity, delamination and stick-slip effects in composites etc. have been treated this way. Within this framework some specialized numerical approximation methods have been developed and tested in structural analysis problems [6-19, 21, 22].

For the treatment of practical structural synthesis (layout) problems, the concept of "structural universe" introduced by Rozvany [2, p. 324; 23, 24] is particularly useful. It is composed by all potential members (components) in a structural design problem and includes every feasible structure, as well as the optimal one. The optimality criteria are used to automatically exclude the unnecessary members, or conversely, to select the members which will form the optimal structure. Optimal layout theory expressed on a structural universe provides a (discretized) approximation of classical Michell's theorem for optimal layout of trusses [27]. The layout of Michell trusses is such as to minimize the total material volume under the assumption of a linear specific cost function for each member. They were the first layout problems solved by automatic, linear programming techniques. We note that in Michell structures equal permissible stresses for tension and compression are assumed and consequently buckling phenomena cannot be considered [9], although they may be critical for some parts of the structure. Thus, a factor that may influence significantly the selection of the optimal layout is omitted if such simplified cost is used. Some recent applications of minimum mass layout of trusses which combine optimality criteria methods, the concept of structural universe, linear programming procedures and iterative refinement techniques are given in Refs $[25,26]$.

In this work we will attempt the integration of Prager's optimality criteria method into the theory of nonsmooth mechanics and its extension to more general cases of cost functions. In Section 2, the theory of optimal structural design is written in a form suitable for further study by means of the nonsmooth mechanics techniques. Then a specific problem concerning the optimal plastic layout of trusses with general convex, nonsmooth and piecewise linear specific cost functions is studied. This application is a generalization of the Michell truss model and an extension through limit analysis techniques of Ref. [14] in the sense that the specific cost functions which are adopted here may have an arbitrary (but finite) number of points of nondifferentiability. Moreover, different permissible stresses in tension and compression can easily be included, thus making possible the incorporation of buckling and limited tension strength effects (important for steel and concrete or masonry structures, respectively).

In Section 5 an iterative algorithm of the sequential linear programming type will be proposed for the numerical treatment of the problem and implementation issues will be discussed. The efficiency of the method is illustrated by characteristic examples in Section 6. Parametric numerical investigation of an optimal truss layout problem permits the discussion of certain aspects of the model which are of particular interest to the structural engineers.

\section{OPTIMAL PLASTIC DESIGN VIA OPTIMALITY CRITERIA}

The static-kinematic optimality criteria method and the related Prager--Shield theory [1] of optimal plastic design will be outlined in this section and its connection to the theory of nonsmooth mechanics will be studied. For the sake of brevity our interest 
will be restricted to the study of optimal plastic design problems on the assumption that the specific cost function for each element of the structure can be expressed as a convex, generally nonsmooth function of the stress components at the same point or the stress of the cross-section in the case of skeletal structures [2].

Let $\psi(x)$ denote the specific cost, i.e. the measure of cost per unit length, area or volume at a point $x$ of the structure and let us assume that $\psi(x)$ depends only on the stress components vector $\mathbf{s}(\mathbf{x})$ (or the vector of generalized stresses) at the same point $x$ :

$$
\psi=\psi(\mathbf{s}(\mathbf{x}))
$$

The stress vector components will be considered as the design parameters of the optimal design problem. The total cost $\phi$ of a structure, which occupies a region $\Omega$ of the Euclidean space $R^{n}(n=1,2$ or 3$)$ in its undeformed state, is obtained by integration:

$$
\Phi=\int_{\Omega} \psi(\mathbf{s}) \mathrm{d} \Omega
$$

In eqn (2) it is implicitly assumed that the problem is one with distributed parameters, i.e. it is assumed that in the search for an optimal structure the adoption of a design with freely varying design parameters is both feasible and costless.

Let $S_{\text {ad }}$ be the space of statically admissible stress distributions of the structure, $\left\{P^{\mathrm{S}}, \mathbf{s}^{\mathrm{S}}\right\}$ be the statically admissible load stress vectors (i.e. they satisfy equilibrium conditions in $\Omega$ and static boundary conditions on its boundary $\left.\Gamma_{p}\right), U_{a d}$ the space of the kinematically admissible displacement distributions and $\left\{\mathbf{u}^{\mathrm{K}}, \mathrm{e}^{\mathrm{K}}\right\}$ be a pair of corresponding kinematically admissible displacements and strains (i.e. they satisfy compatibility conditions in $\Omega$ and kinematic boundary conditions on $\Gamma_{\mathrm{u}}$ ). $\Gamma_{\mathrm{p}}$ (respectively $\Gamma_{\mathrm{u}}$ ) denotes the part of the boundary $\Gamma$ of the structure where loading (respectively displacements) is prescribed and $\Gamma_{\mathrm{u}} \cup \Gamma_{\mathrm{p}}=\Gamma, \Gamma_{\mathrm{u}} \cap \Gamma_{\mathrm{p}}=0$ hold.

For a given stress-strain law for the material of the structure (constitutive law), the structural analysis problem consists of the determining a distribution of statically admissible stresses $\mathbf{s}^{\mathbf{s}}$ and kinematically admissible strains $e^{k}$, which also satisfy the constitutive law at each point of the structure.

On the other hand the lower bound (static) theorem of plastic limit analysis ensures that every statically admissible stress field furnishes a lower bound on the true ultimate load capacity of the structure (see e.g. Refs $[28,29]$ among others). Therefore if a certain statically admissible stress field for a given structure is considered, the dimensioning of the structure (e.g. cross-section parameters) can be defined from the requirement that the yield inequality of plasticity should be satisfied everywhere, thus providing a safe plastic design.
If, in addition we consider the optimality requirement, the choice of the statically admissible stress field in the plastic design procedure must also lead to a minimum value of the total cost of the structure (2). Thus the following problem arises.

Problem 1. Find a stress field $\mathbf{s}^{\mathrm{s}} \in S_{\mathrm{ad}}$ minimizing the total cost $\Phi$ of the structure:

$$
\min \Phi=\left\{\min \Phi=\int_{\Omega} \psi\left(\mathbf{s}^{\mathrm{s}}\right) \mathrm{d} \Omega / \mathbf{s}^{\mathbf{5}} \in S_{\mathrm{ad}}\right\}
$$

At the solution of Problem 1 the following static-kinematic optimalily conditions must hold $[1,2$, p. 38$]$.

Theorem 1. Find a kinematically admissible strain field $\mathrm{e}^{\mathrm{k}}$ and a stress field $\mathrm{s}^{\mathrm{s}} \in S_{\mathrm{ad}}$, such that the following relation holds at all points of the structure:

$$
\mathbf{e}^{\mathrm{K}}=\mathbf{G}\left\{\psi\left(\mathbf{s}^{\mathbf{s}}\right)\right\} \text { on } \Omega
$$

where $G$ denotes an appropriate generalization of the gradient operator.

Outline of the proof $[1,2$, p. 40$]$. Let $\psi$ in eqn (1) be identified with the specific complementary energy of a fictitious nonlinear elastic structure with the same boundary conditions and loading as the one studied (to be called "associated" structure in the following). By definition the nonlinear constitutive (strain -stress) law of the fictitious structure is given by the relation:

$$
\mathbf{e}=\frac{\partial \psi}{\partial \mathbf{s}}
$$

where for demonstration smooth differentiable functions $\psi$ are assumed. The complementary energy theorem for the associated structure leads to the following conclusion: the total cost $\Phi$ of the plastic system and the total complementary energy of the associated elastic system attain their minimum simultaneously when the strain-stress relation (5) and kinematic admissibility are satisfied. Thus the optimality conditions of Theorem 1 arise for the case of smooth specific cost functions $\psi$. The generalization to the wider class of nonsmooth functions is straightforward [6, 20]. q.e.d.

It must be noted here that the cost minimality condition (4) is a necessary and sufficient condition for the case of convex specific cost functions and linear stress equilibrium and strain compatibility relations (i.e. for first-order structural analysis theory with small displacements and deformations). In any other case and especially for nonconvex specific cost functions, only condition (4) is necessary $[2,9$, p. 65].

Theorem I permits us to study the nonlinear associated structural analysis problem instead of the original optimal structural design problem. To this end certain results from the theory of nonsmooth 
mechanics will be used $[3-6,16]$. We observe that the constitutive law (4) can be derived by means of generalized differentiation $G$ from a potential function $\psi(\mathbf{s})$, called superpotential because it may be a general nonsmooth and nonconvex function. The appropriate generalization of the differential operator $G$ which must be used in eqn (4) depends on the nature of the specific cost function $\psi(\mathbf{s})$. The following cases require different definitions of $G$ :

(1) for a smooth function $\psi$ (i.e. a $C^{1}$ function) $G$ is the classical differential operator, thus eqn (4) simplifies to

$$
e=\frac{d \psi}{d s}
$$

(2) for a convex, continuous but generally nonsmooth function $\psi$ (i.e. a $C^{0}$ function) $G$ is the subdifferential operator $\hat{c}$ of convex analysis [4] defined by

$$
\begin{aligned}
\mathbf{e} \in \hat{\partial} \psi(\mathbf{s})=\left\{\mathbf{e}^{*} /\left\langle\mathbf{e}^{*}, \mathbf{s}^{*}-\mathbf{s}\right\rangle\right. & \\
& \left.\leqslant \psi\left(\mathbf{s}^{*}\right)-\psi(\mathbf{s}), \quad \forall \mathbf{s}^{*} \in S_{a d}\right\},
\end{aligned}
$$

where $\langle.,$.$\rangle denotes the inner product between the$ energy corresponding quantities $\mathbf{e}$ and $\mathbf{s}$; in this case the strain-stress law of eqn (4) is a monotone function with complete vertical ascending branches (multivalued function or multifunction);

(3) for a nonconvex, Lipschitz continuous but generally nonsmooth function an appropriate generalization of the subdifferential operation of convex analysis must be considered in the place of $G$. For instance the generalized subdifferential operator of Clarke or the quasidifferential operator in the sense of Demyanov may be used here $[3,4,6,16,17,21,31]$.

Nonconvex specific cost functions fall outside the scope of this paper and thus they will not be studied further (the interested reader is referred to the above cited publications). Moreover, the treatment of problems with smooth specific cost functions case 1 is trivial. Our interest will be focused on convex and nonsmooth specific cost functions (case 2). This case includes a fairly general class of specific cost functions which arise from models with an optimal selection mechanism. The feature of switching between different design strategies is essential for the optimal design model and may lead to important material savings.

A characteristic example of convex and nonsmooth specific cost function is the max-type function: we assume that in the course of designing a certain structural member $m$, different design concepts can be applied. Each of them may include different choice of materials, cross-sections, construction methods etc. We assume further that the cost of the $j$ th design is modeled by a convex (possibly linear) specific cost function $\psi_{j}(\mathbf{s}), j=1 \cdots m$. Therefore a composite specific cost function (Fig. 1a),

$$
\psi(\mathbf{s})-\max _{j=1 \cdots m} \psi_{j}(\mathbf{s})
$$

would give to the optimal design model the ability to choose automatically between different design technologies. Other examples of specific cost functions will be considered in Section 5.

\section{A VARIATIONAL INEQUALITY APPROACH TO THE STATIC ANALYSIS OF STRUCTURES WITH COMPLETE STRESS-STRAIN LAWS}

The optimality criteria method which was outlined in the previous paragraph transforms the optimal design problem into an equivalent nonlinearly elastic structural analysis problem for a structure with a specific complementary energy function which is eyual to the specific cost function of the initial optimization model. The structural analysis problem that is associated with the initial optimal design problem leads to the formulation of a pair of variational inequalities and the corresponding convex minimization "principles".

On the assumption that the classical stress equilibrium and strain compatibility relations of the linearized elasticity hold and the material constitutive
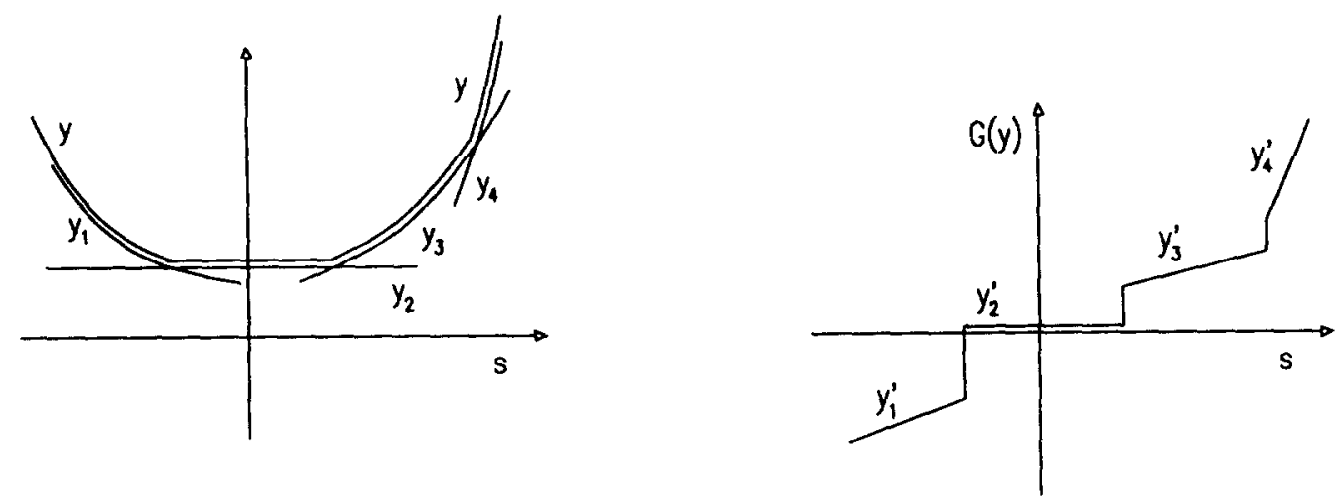

Fig. 1. A convex, nonsmooth specific cost function of max type and the corresponding optimality criteria. 
law (strain-stress relation) is given by eqn (7), the structural analysis problem reads $[3,6]$ :

Problem 2. Find a stress field $\mathbf{s} \in S_{\mathrm{ad}}$ satisfying the variational inequality

$$
\int_{\Omega} \psi(\mathbf{t}) \mathrm{d} \Omega-\int_{\Omega} \psi(s) \mathrm{d} \Omega \geqslant \int_{\Gamma}\langle\mathbf{u}, \mathbf{t}-\mathbf{s}\rangle \mathrm{d} \Gamma, \quad \forall \mathbf{t} \in S_{\mathrm{ad}} .
$$

An equivalent minimization problem for the complementary energy of the structure, which is a convex and nonsmooth function, has the form:

Problem 3. Find a stress field $\mathrm{s} \in S_{\mathrm{ad}}$ minimizing the complementary energy of the structure:

$$
\Pi^{\mathrm{c}}(\mathbf{s})=\left\{\min \Pi^{\mathrm{c}}(\mathbf{t}) / \mathbf{t} \in S_{\mathrm{ad}}\right\}
$$

with

$$
\Pi^{\mathrm{c}}(\mathbf{t})=\int_{\Omega} \psi(\mathbf{t}) \mathrm{d} \Omega+\int_{\Gamma_{\mathrm{u}}}\langle\mathbf{u}, \mathbf{t}\rangle \mathrm{d} \Gamma_{\mathbf{u}} .
$$

The main variables in problems 2 and 3 are the stresses which must belong to the set of statically admissible stresses $S_{\text {ad }}$. The solution of either problem requires the application of a force-type structural analysis technique, a fact which is not advantageous from the computational point of view. Therefore we will reformulate problems 2 and 3 in the displacement method form, which at present is more familar to structural analysts.

From the convex specific cost function (1) we derive its complementary cost function:

$$
\hat{\psi}(\mathbf{e})=\max \{\langle\mathbf{e}, \mathbf{s}\rangle-\psi(\mathbf{s})\},
$$

where $\hat{\psi}$ is the convex conjugate function of $\psi(\mathbf{s})$, $\left(\hat{\psi}=\psi^{\mathfrak{c}}\right)[6,30]$.

Analogously to eqn (2) the total complementary cost function of the structure, which will be identified with the potential energy of the associated structure, is expressed by:

$$
\hat{\Phi}=\int_{\Omega} \hat{\psi}(\mathbf{e}) \mathrm{d} \Omega .
$$

Definitions (12) and (13) and classical convex duality theory $[6,30,33]$ lead to the following reformulation of problems 1-3.

Problem 1'. Find a displacement field $\mathbf{u}^{\mathrm{K}} \in U_{\text {ad }}$ maximizing the total complementary cost of the structure:

$$
\max \hat{\boldsymbol{\Phi}}=\left\{\max _{\mathbf{e}^{\mathrm{K}, \mathrm{u}^{\mathrm{K}}}} \hat{\boldsymbol{\phi}}=\int_{\Omega}\left\{\left\langle\mathbf{u}^{\mathrm{K}}, \mathbf{p}\right\rangle-\hat{\psi}\left(\mathbf{e}^{\mathrm{K}}\right)\right\} \mathrm{d} \Omega\right\} .
$$

The Prager-Shield static-kinematic optimality criteria are written accordingly $[3,6]$ :

$$
\mathbf{s}^{\mathbf{s}} \in \partial \hat{\psi}\left(\mathbf{e}^{\mathrm{K}}\right) \text { on } \Omega
$$

and the associated structural analysis problem takes the following form [note that $-\hat{\phi}$ of eqn (13) is the total potential energy of the structure].

Problem 2'. Find a kinematically admissible displacement field $\mathbf{u}^{\mathrm{K}} \in U_{\text {ad }}$ such as to satisfy the variational inequality:

$$
\begin{aligned}
\int_{\Omega} \hat{\psi}(\mathbf{e}(\mathbf{v})) \mathrm{d} \Omega & -\int_{\Omega} \hat{\psi}(\mathbf{e}(\mathbf{u})) \mathrm{d} \Omega \\
& \geqslant \int_{\Gamma_{\mathrm{p}}}\langle\mathbf{p}, \mathbf{v}-\mathbf{u}\rangle \mathrm{d} \Gamma, \quad \forall \mathbf{v} \in U_{\mathrm{ad}},
\end{aligned}
$$

where $\mathbf{e}(\mathbf{u})$ denotes the linear kinematic compatibility operator. In terms of potential energy the following problem is then formulated.

Problem 3'. Find the displacement field $\mathbf{u}^{\mathrm{K}} \in U_{\mathrm{ad}}$ minimizing the potential energy of the structure:

$$
\Pi(\mathbf{u})=\left\{\min \Pi(\mathbf{v}) / \mathbf{v} \in U_{\mathrm{ad}}\right\}
$$

with

$$
\Pi(\mathbf{v})=\int_{\Omega} \hat{\psi}(\mathbf{e}(\mathbf{v})) \mathrm{d} \Omega-\int_{\Gamma_{\mathrm{p}}}\langle\mathbf{p}, \mathbf{v}\rangle \mathrm{d} \Gamma_{\mathrm{p}} .
$$

Problems 2, 3 and 2', 3' have been studied extensively in the nonsmooth mechanics literature $[3-6,18]$ and certain algorithms cxist for their numcrical trcatment $[6,15,17,19,21,22]$. In Section 5 the form (17) -(18) of Problem 3 will be used for the formulation of a numerical algorithm which takes advantage of the piecewise linear nature of the specific cost function $\hat{\psi}$.

\section{OPTIMAL LAYOUT PROBLEMS AND THE CONCEPT OF STRUCTURAL UNIVERSE}

Problems of optimal layout arise when one considers not only the optimal selection of cross-sections and/or the optimal position of some nodes of the structure for a given structural layout (shape, connectivity etc.), as was the case in Section 2 , but also the more general question of the optimal choice of topology, in the sense of the spatial sequence and the connectivity of the members and joints.

Following a general scheme introduced by Rozvany $[2,9]$ for the study of optimal layout problems, we restrict our objective by introducing the concept of the "structural universe", mentioned in the Introduction: the search is restricted in the set of elements belonging to a predefined set of candidate members that constitutes a (discretized) approximation of the physical space the structure is permitted to cover.

The structure to be optimized must be able to transfer a given system of loads to a given support boundary $\Gamma^{\prime}$ (or part of it) subject to some constraints on the available space $\left(\Omega^{\prime}\right)$. The whole or part of $\Omega^{\prime}$ is covered by a grid of nodes, which includes the 
support boundaries and the load application points. Then a number of potential structural members is introduced connecting the nodes in an arbitrary pattern (some restrictions on the pattern selection will be discussed in Section 5). The set of all elements ( $m$ in total) that are used for the composition of all possible statically determinate and rigid structures constitutes the structural universe, within which lie all the feasible designs (alternatively the term "ground structure" is proposed in Ref. [23, Section 3]). The structural layout problem is now restricted to structures which are composed of members of the structural universe.

The Prager -Shield optimal design theory for structures with given topology is subsequently applied on the structural universe. The static-kinematic optimality criteria of this theory result in zero stresses for some potential structural members. Since those elements do not contribute to the load transfer, they can be omitted without consequence, provided that the remaining stressed members do not have kinematic mobility (i.e. they do not form a mechanism). This idea guides the search for an optimal structure composed of selected elements within the structural universe.

This methodology for the treatment of the optimal layout problem avoids complications which are connected with other optimal layout approaches and makes the incorporation of the layout problem in the general nonsmooth mechanics approach far easier. We note that the general optimal design problem with variable position of the nodes and variable connectivity of the structural elements at the same time, leads generally to be a nonconvex problem. In contrast, for the same problem defined over a structural universe, convex spccific cost functions lead to convex associated elastostatic analysis problems.

The optimality of the best layout which can be constructed by taking members from a particular structural universe is restricted by grid coarseness and the pattern assumed for the potential structural components (cf. Ref. [32, Section 12.3.4], concerning bounds of the continuous solution and Ref. [25] for numerical examples in the case of a simple truss). An intermediate solution which has been obtained with a certain discretization can be ameliorated either by refining the mesh or increasing the density of the element's pattern on the whole or selected parts of $\Omega^{\prime}[25,26]$ (the best strategy clearly depends on the particular problem treated and the arguments for either treatment are probably similar to those in the discussion on mesh refinement compared to element complexity increased in finite element problems).

\section{A SEQLENTIAL LINEAR APPROXIMATION ALGORITHM FOR OPTIMAL LAYOUT PROBLEM}

In this section the theory is applied to a model problem which concerns the optimal layout of plane trusses. The specific cost function $\psi_{i}$ for such struc- tural components of the truss $i=1 \cdots m$ is a convex, piecewise linear function of the axial force $N_{i}$ of the member. The cost of the structural connections and the supports are assumed negligible.

In the simplest case the specific cost function is written (Fig. 2a):

$$
\psi_{i}\left(N_{i}\right)=k_{1}\left|N_{i}\right|^{+}-k_{2}\left|N_{i}\right|^{\cdots}
$$

where $|x|^{+}=|x|+x / 2,|x|^{-}=|x|-x / 2$ denote the positive and negative part of a real variable $x$, respectively, and $k_{1}$ (respectively $k_{2}$ ) is given nonnegative constants indicating the cost per unit positive (respectively negative) axial force transmittcd by the structural member on hand. Note that eqn (19) can be derived by the general form of eqn (8) with $\psi_{1}=k_{1} N$ and $\psi_{2}=-k_{2} N$.

The complementary specific cost function $\hat{\psi}_{i}(12)$ of eqn (19) is (Fig. 2b):

$$
\begin{aligned}
& \hat{\psi}_{i}\left(\epsilon_{i}\right)=[0,+\infty), \quad \epsilon_{i}=k_{1} \\
& \hat{\psi}_{i}\left(\epsilon_{i}\right)=0, \quad-k_{2}<\epsilon_{i}<k_{1} \\
& \hat{\psi}_{i}\left(\epsilon_{i}\right)=[0,+\infty), \quad \epsilon_{i}=-k_{2} .
\end{aligned}
$$

The generalized stress-strain law to be used for the nonlinear structural analysis of the associated elastic system is derived from the Prager-Shield optimality condition (15) that may be written explicitly for this case (Fig. 2d):

$$
\begin{aligned}
& N_{i} \geqslant 0, \quad \text { for } \epsilon_{i}=k_{1} \\
& N_{i}=0, \quad \text { for }-k_{2}<\epsilon_{i}<k_{1} \\
& N_{i} \leqslant 0, \quad \text { for } \epsilon_{i}=-k_{2} .
\end{aligned}
$$

Owing to the generality of the formulation of Sections 2 and 3 [cf. definitions (4), (7) and (15)], the simple cost of Fig. 2 a can be replaced by the piecewise linear function of Fig. 3a without any modification of the model. The conjugate cost function $\hat{\psi}$ and the corresponding optimality criteria are depicted in Fig. $3 \mathrm{~b}$ and $\mathrm{c}, \mathrm{d}$, respectively.

In this case the cost per unit member force (equivalently the slope of each linear branch of the piecewise linear cost function) is different at various force levels. Use of various materials or structural element configurations (i.e. a heavier construction that takes into account buckling restrictions for compressive element forces) leads to generally piecewise smooth nonlinear cost functions. The points of nondifferentiability correspond to force levels where a choice between alternative material or design configurations must be made. The optimum of the alternative design concepts is found automatically by the model analyzed in this paper. For reasons of clarity, we assumed here a piecewise linear cost function for the model problem which is treated in this section and in 


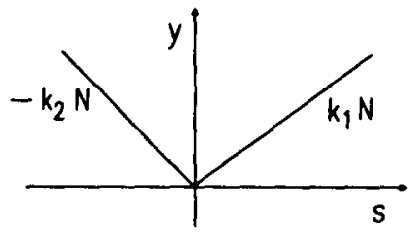

(a)

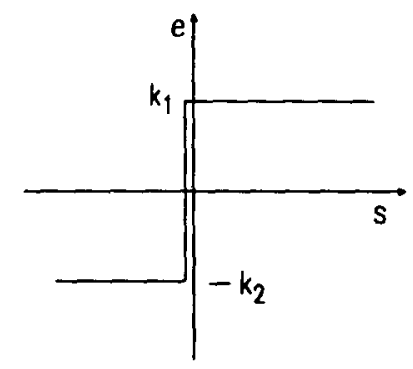

(c)

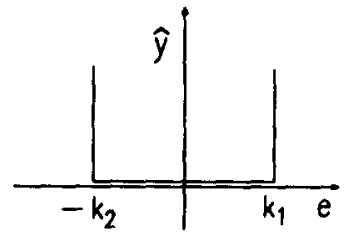

(b)

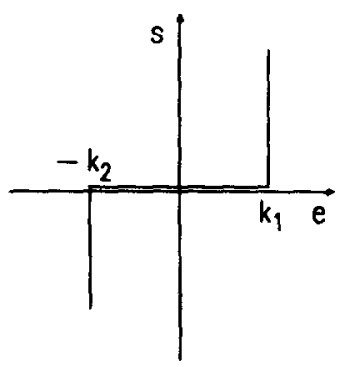

(d)

Fig. 2. Michell-Prager-Shield specific cost function $\psi(\mathbf{s})$, complementary specific cost function $\psi(\mathbf{e})$ and the corresponding static-kinematic optimality conditions.

the numerical examples. However, the theory which was developed in the previous sections is valid for any piecewise continuous and convex cost function.

After performing a discretization of the physical space, a structural universe is defined according to the previous section, such as not to restrain (worsen) umnecessarily the solution. Let $n$ be the number of nodes and $m$ be the number of potential elements included in the structural universe. Then the optimal solution is defined from the potential energy minimization problem $3^{\prime}$ of the associated structure (17-18):

$$
\min _{\mathbf{v} \in U_{\mathrm{ad}}} \Pi(\mathbf{v})=\sum_{i=1}^{m}\left(\hat{\psi}_{i}(\mathbf{e}(\mathbf{v}))\right)-\mathbf{p}^{\mathrm{T}} \mathbf{v}
$$

The existence of nondifferentiability points in the range of the functionals $\hat{\psi}_{i}$ involved in the definition of $\Pi$ makes impossible the application of classical nonlinear minimization methods that are based on a smoothness assumption. For that reason an active set-type procedure is introduced that is able to account for the nonsmooth sections of the domain.

Due to the existence of the nonsmoothness points and the piecewise linearity, the functions $\bar{\psi}_{i}$ can be alternatively written in the form of a max-type function: $\hat{\psi}_{i}(\mathbf{v})=\max _{i=1 \ldots k}\left\{\hat{\psi}_{i j}(\mathbf{v})\right\}, i=1 \cdots m$, where $\hat{\psi}_{i j} \mathrm{~s}$ are affine functions (see Fig. 1).

The nonsmooth unconstraincd minimization problem (22) is then transformed to an equivalent constrained smooth minimization problem by a technique that is common when the max-type functionals are involved. An auxiliary vector of variables $\phi \in R^{m}$ is introduced (having the meaning of the eipigraphs of $\hat{\psi}_{i}$ s) and problem (22) is reformulated to:

$$
\min _{\mathbf{v} \in U_{\mathrm{ad}}, \phi \in R^{m}} \Pi(\mathbf{v}, \phi)=\sum_{i=1}^{m} \phi_{i}-\mathbf{p}^{\mathrm{T}} \mathbf{v}
$$

subject to

$$
\begin{aligned}
\phi_{i} \geqslant \hat{\psi}_{i j} \Leftrightarrow c_{i j}=\phi_{i}-\hat{\psi}_{i j} & \geqslant 0, \\
i & =1 \cdots m, \quad j=1 \cdots k .
\end{aligned}
$$

At every point within the domain of eqn (23) (and at the minimum also), only some of the constraints (24) are satisfied as equalities (at most two for every bar element $i$ in the case of the truss model). This fact is used to replace the inequality constrained linear program (23)-(24) by another that has fewer equality constraints and thus is simpler to solve.

We introduce the set $M$ of constraint indexes that are active at the current point in the solutions space: $M_{i}=\left\{j: c_{i j}=0, j=1 \cdots k\right\}$. At every point $\mathbf{v}^{k} \in U_{\text {ad }}$, $M_{i}$ s have at least one element $q_{i}$ (called "primary") that is used to eliminate the corresponding auxiliary variables $\phi_{i}$. Then a local approximation of problem (23)-(24) is:

$$
\min \mathbf{r}^{\mathrm{T}} \mathbf{d}+c,
$$




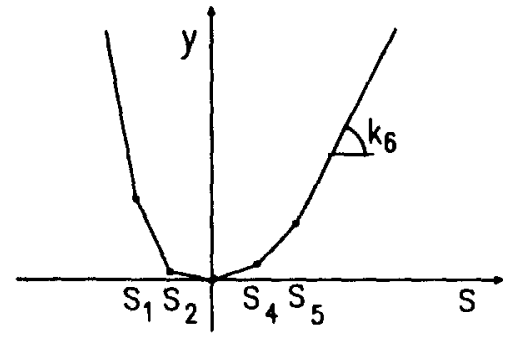

(a)

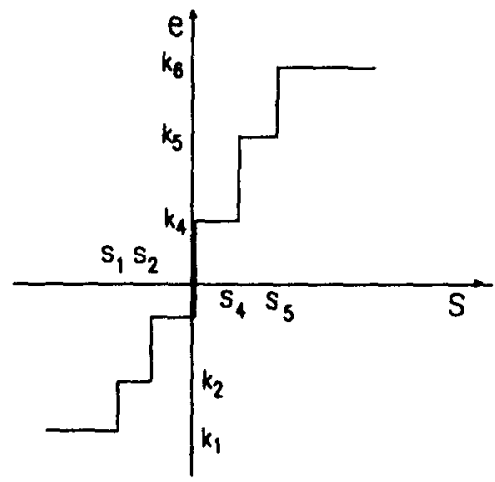

(c)

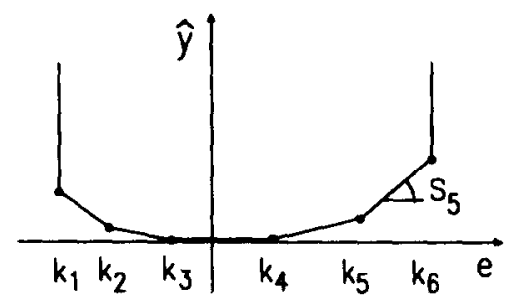

(b)

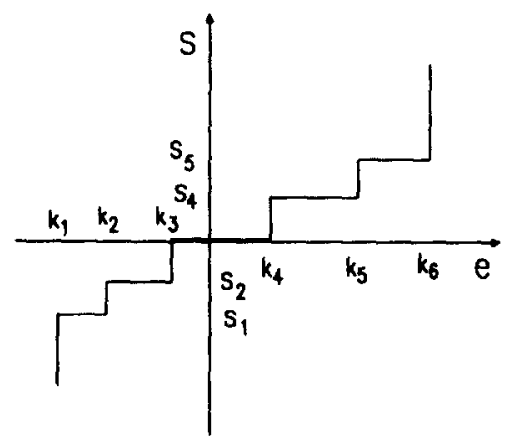

(d)

Fig. 3. Specific cost function $\psi(\mathbf{s})$, complementary specific cost function $\hat{\psi}(\mathbf{c})$ and the corresponding static-kinematic optimality conditions.

subject to

$$
\mathbf{A}^{\mathrm{T}} \mathbf{d}=\mathbf{b},
$$

where $\mathbf{r}=\sum_{i=1}^{m} \hat{\psi}_{i q i}^{\prime}-\mathbf{p}, \mathbf{A}_{i i}=\hat{\psi}_{i i}^{\prime}-\hat{\psi}_{i g i}^{\prime}, \mathbf{b}_{i}=\hat{\psi}_{i j}\left(\mathbf{v}^{k}\right)-$ $\hat{\psi}_{i q i}\left(\mathbf{v}^{k}\right), c=\sum_{i=1}^{m} \hat{\psi}_{i q i}\left(\mathbf{v}^{k}\right)$ and $\mathbf{d}=\mathbf{v}^{*}-\mathbf{v}^{k}$ is the predicted step to the minimum $\mathbf{v}^{*}$.

Problem (25)-(26) constitutes a local approximation of the nonsmooth superpotential $\Pi$ of eqn (22) at $\mathbf{v}^{k}$ by a piecewise affine functional. If the active set is correct, then each constraint in eqn (26) indicates the position of a "groove" separating two adjoint smooth parts of $\Pi$, along which the classical derivative of $\Pi$ is discontinuous and the introduction of the subdifferential becomes necessary (cf. [17, Chap. 4, 22, 34, Chap. 14] for a detailed analysis of the approximation method).

The solution of problem (22) is thus reduced to a sequence of intermediate linear programming steps:

(1) Initialization: $k=1, v^{k}=0 M_{i}^{k}=\left\{q_{i}^{k}\right\}$, where $q_{i}^{k}\left(\mathbf{v}^{k}\right)$ is one of the indexes active at $\mathbf{v}^{k}$ (in the sense that $c_{i q i}=0$ or equivalently $\hat{\psi}_{i}=\hat{\psi}_{i q i}$ ).

\section{(2) Step computation:}

- select primary indexes $q_{i}^{k} \in M_{i}^{k}$;

- compute the function gradient $\mathbf{r}$ and the coefficients A, b of constraints (26) (one constraint for each element of $M_{i}^{k}-\left\{q_{i}^{k}\right\}$ );

- solve problem (25)-(26) for $\mathbf{d}$ and compute the Lagrange multipliers.

(3) Termination: if $|\mathbf{d}| \leqslant \epsilon$ and all Lagrange multipliers are positive, then accept $\mathbf{v}^{k}$ as a solution to problem (22) and stop.

(4) Active set update:

- set $M^{k+1}=M^{k}, \mathbf{v}^{k+1}=\mathbf{v}^{k}+\mathbf{d}$ and compute new active indexes $q_{i}^{k+1}$;

- augmentation-if $q_{i}^{k+1} \notin M_{i}^{k+1}$, then $M_{i}^{k+1}=$ $M_{i}^{k+1}+\left\{q_{i}^{k+1}\right\}$

- reduction-if $M^{k+1}$ is unchanged then remove the constraint with the largest negative Lagrange multiplier;

- set $k=k+1$ and restart from step 2 . 
The number $t$ of columns in the constraint matrix A equals the total count of active constraints reduced by $m$ (primarics). It can be casily seen that $\mathbf{A}$ has the structure of the equilibrium matrix of the truss with the nonzero entries positioned within a diagonal band. Consequently, the parameters of problem (25) -(26) can be stored and treated (using specialized linear programming solvers [36, Section 5.6]) in computational space linear to the problem's leading dimension $n$. This is an important property since it is making possible the solution of practical layout problems of large dimension (or equivalently: defined on sufficiently dense structural universes).

The sparsity of A decreases while the "locality" of conections diminishes, in the sense that each node, in addition to its immediate neighbors, is connected to increasingly distant nodes. Thus for computational efficiency the element pattern connecting the initial grid nodes should be local (for the bandwidth of $\mathbf{A}$ to be kept as low as possible), repetitive (for practical automatic generation purposes) and for accuracy of the discrete representation of the structure it should preferably cover the principal directions of the associated displacement field [2, Section 8.3c].

By definition, the generalized cost functions introduced in this paper end in horizontal branches that give rise to vertical branches in the conjugate functions (see Fig. 3c,d). Thus the domain of the potential encrgy $\Pi(22)$ is bounded in all directions (it suffices to be possible to form at least one rigid statically determinate structure using the elements of the initial

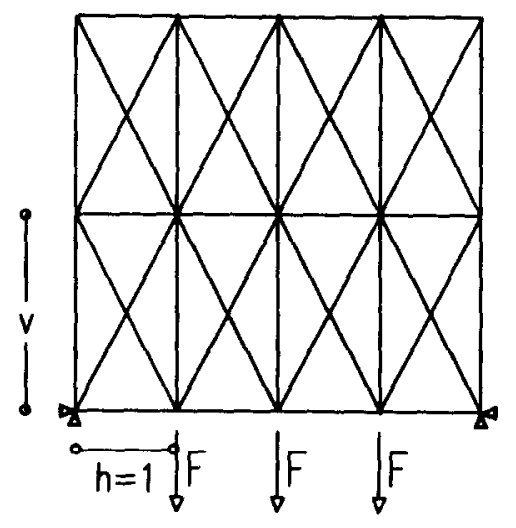

$(0)$

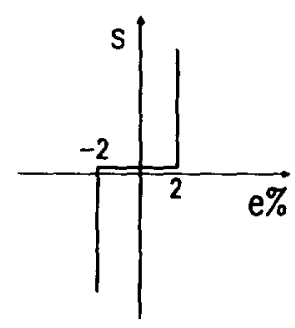

(1)

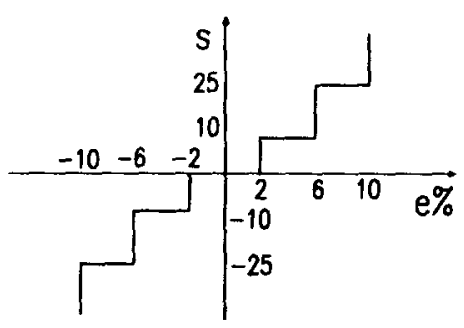

(2)

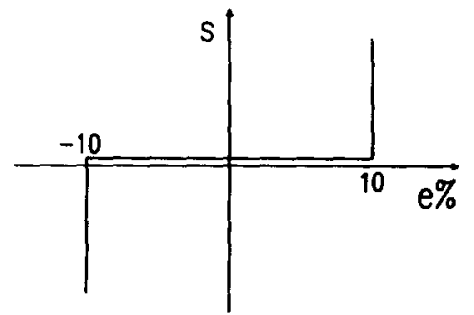

(3)

(b)

Fig. 4. Example of a bridge truss: (a) geometry and loading parameters; (b) optimality criteria used for comparison. 
grid, that is a necessary condition to define the structural universe). Consequently there is no danger of the linear approximation of eqn (25) becoming unbounded in any search direction, which is a common danger in linear programming.

Although there exist other approaches to the treatment of the composite nonsmooth minimization problem (22) (cf. [34, Chap 14] for complete discussion and literature), the active set method described here, more than its excellent local convergence characteristics, has the advantage of maintaining the banded structure of the current subdifferential approximation (matrix A), a property that removes the size limitations and makes the setup and the solution of the subproblems (25)-(26) far easier.

The total cost of the structure at the solution can be computed directly using the definition (3). Alternatively, relation (14) can be employed since the integral of the conjugate cost may easily be computed during the solution of each subproblem [partly contained in the constant term $c$ of eqn (25)]. In the present form of the algorithm, the latter approach was preferred since it is nearly costless.

The optimal plastic truss layout method described as a model problem is directly applicable to three-dimensional truss problems.

It can also be applied to other optimal plastic design and layout problems for two- and three-dimensional structures (e.g. plates, shells), as far as they can be modeled by means of Hrenikoff's truss model. Furthermore, the theory of Sections 2 and 3 is valid for general two- and three-dimensional optimal layout problems.

\section{NUMERICAL RESULTS AND DISCUSSION}

To illustrate the effectiveness of the approach described in the previous sections we studied the optimal design of a plane bridge truss. This simple case was preferred since it has already been the subject of similar studies [23] and thus qualitative verification of the results is possible.

The position of the supports and the loads are depicted in Fig. 4a. Note that the vertical grid dimension and the load are variable $\left(F=\lambda[0,-5]^{\mathrm{T}}\right)$. A simple bar pattern is used for the construction of the structural universe covering the expected principal stress directions. The pattern selected is strictly local (i.e. a node is connected only with its neighbors) in contrast to the one used in Ref. [23].

For constant vertical dimension $v=2$ the three alternative optimality criteria were tried for comparison, numbered 1, 2 and 3 (Fig. 4b), corresponding to decreasing yield limits (or equivalently progressively increasing values of $\epsilon$ showing the position of the highest/lowest horizontal branch). The first and last criteria are of the classical type encountered in previous studies, while the second criterion contains multiple horizontal and vertical branches, both in tension and compression.
Cases 1 and 3 correspond to "simple" (e.g. one material) designs with a cost per unit force equal to 2 and 10 , respectively, while case 2 corresponds to a more flexible design (e.g. a number of materials are available from which the designer will choose) where the cost per unit force takes the values 2,6 and 10 in the intervals $\|S\| \leqslant 10,10 \leqslant\|S\| \leqslant 25$ and $25 \leqslant\|S\|$, respectively.

In the first group of trials the vertical load parameter $\lambda$ was varied in the range $(1,20)$. The effect of using general, more flexible design concepts (i.e. cost functions) is examined. The resulting total cost values are depicted in Fig. 5, together with the configurations of the structure at the points where the layout is changing. As is to be expected, criterion 2 results in higher cost than criterion 1 (corresponding to material with higher yield limit). The gain from the use of a design based on case 1 tends to decrease for the lowest load values. In contrast, it provides invariably "cheaper" solutions than criterion 3 (up to $75 \%$ lower in the worst case). On the other hand certain factors which are not included in the weight optimization problem treated here may be critical in some applications. For instance we observe that, for the whole range of $\lambda$, the total length of the bars of case 2 is greater than either one of the other cases.

In the second set of trials the load parameter was kept constant $\dot{\lambda}=7$ and we varied the vertical grid dimension $v$, which resulted in changes of the grid aspect ratio $(v / h)$ in the range $(0.25,2.5)$. The effect of the geometry of the structure on the total cost is examined. In Fig. 6 are shown the resulting cost values for criteria 2 and 3 and the corresponding structure configurations. Note the characteristic abrupt cost decrease for value of the aspect ratio lower than 0.50 caused by the change in the load transfer mechanism from that of an arch to a beam, the latter one being significantly better between the examined configurations.

All the trials were performed using the Harwell code LA01 for solving the linear programming subproblem. This solver is not taking advantage of the

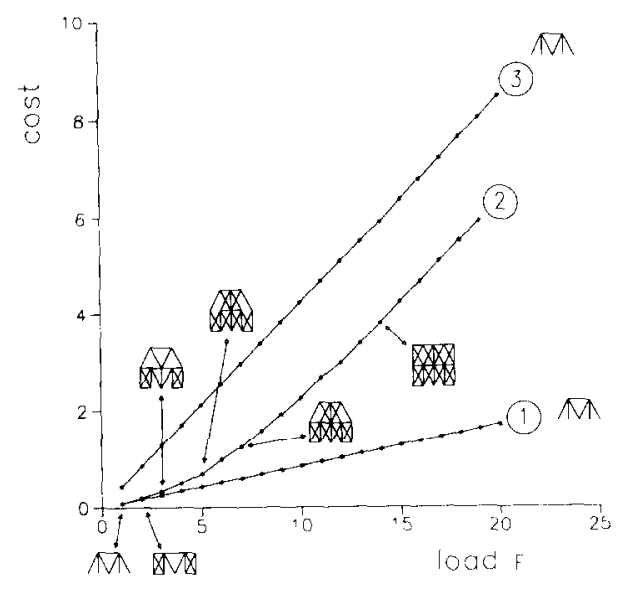

Fig. 5. Cost variation with load. 


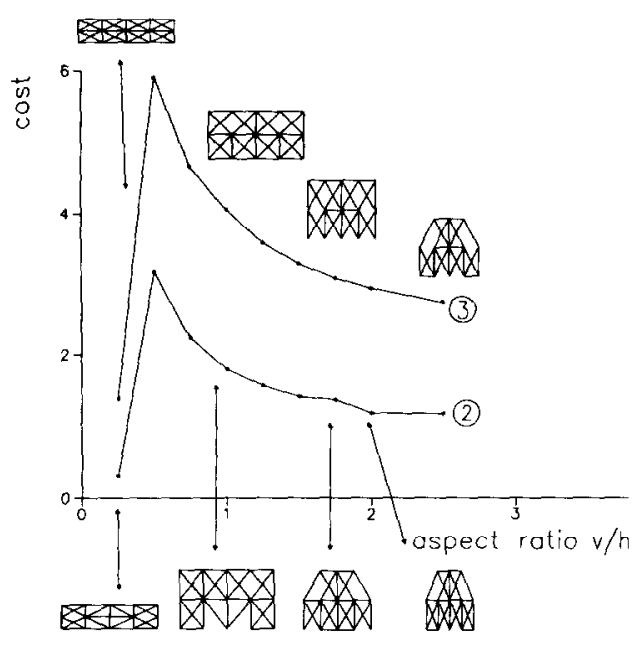

Fig. 6. Cost variation with aspect ratio.

constraints matrix sparsity and thus it was expected to magnify any instabilities of the approximation model. Indeed, some precision problems encountered in the early applications were easily corrected by proper scaling. Recently the linear subproblem solver has been replaced by the set of SPLO routines that are able to treat large sparse problems [35]. Preliminary results verified the previous computations and, as it was expected, indicated significant savings in computational memory and time requirements as the problem dimension increased. Extensive testing of the method in large scale problems is considered necessary and it will be the subject of forthcoming research.

Acknowledgements--The authors would like to express their gratitude to Professor P. D. Panagiotopoulos, Aristotle University, Thessaloniki, Greece and RWTH Aachen, Germany for his continuous encouragement and support. The second author was supported by the Alexander von Humboldt Stiftung.

\section{REFERENCES}

1. W. Prager and R. T. Shield, A general theory of optimal plastic design. J. Appl. Mech. 34(1), 184-186 (1967).

2. G. I. N. Rozvany, Structural Design via Optimality Criteria. The Prager Approach to Structural Optimization. Kluwer Academic, Dordrecht (1989).

3. J. J. Moreau, P. D. Panagiotopoulos and G. Strang (eds), Topics in Nonsmooth Mechanics. Birkhäuser, Boston (1988)

4. J. J. Moreau and P. D. Pangiotopoulos (eds), Nonsmooth Mechanics and Applications. CISM Lecture Notes 302, Springer, Wien (1988).

5. P. D. Panagiotopoulos, A variational inequality approach to the inelastic stress-unilateral analysis of cable structures. Comput. Struct. 6, 133-139 (1976).

6. P. D. Panagiotopoulos, Inequality Problems in Mechanics and Applications. Convex and Nonconvex Energy Functionals. Birkhäuser, Boston (1985). [Russian translation by Mir, Moscow (1989).]

7. E. A. Sadek, Direct versus indirect methods in structural optimization. Comput. Struct. 23, 725-737 (1986).

8. E. A. Sadek, Optimization of structures having general cross-sectional relationships using an optimality criterion method. Comput. Struct. 43, 959-969 (1992).
9. G. I. N. Rozvany, Optimal Design of Flexural Systems: Beams, Grillages, Slabs, Plates and Shells. Pergamon Press, New York (1976).

10. G. Strang and R. V. Kohn, Optimal design in elasticity and plasticity. Int. J. Numer. Meth. Engng 22, 183-188 (1986).

11. R. H. Hill and G. I. N. Rozvany, Prager's layout theory: a nonnumeric computer method for generating optimal structural configurations and weight influence surfaces. Comput. Meth. Appl. Mech. Engng 49(1), 131-148 (1985).

12. J.-M. Lagache, A geometrical procedure to design trusses in a given area. Engng Opt. 5(1), 1-12 (1980).

13. G. I. N. Rozvany and R. H. Hill, A computer algorithm for deriving analytically and plotting the optimal structural layout. Comput. Struct. 10(1), 295-300 (1979).

14. G. Strang and R. V. Kohn, Hencky-Prandtl nets and constrained Michell trusses. Comput. Meth. Appl. Mech. Engng 36, 207-222 (1985).

15. H. Antes and P. D. Panagiotopoulos, An Integral Equation Approach to the Static and Dynamic Contact Problems. Equality and Inequality Methods. Birkhäuser, Boston (1992).

16. G. E. Stavroulakis, Convex decomposition for nonconvex energy problems in elastostatics and applications. Eur. J. Mech. A, Solids 12(1), 1-20 (1993).

17. M. Ap. Tzaferopoulos, Numerical analysis of structures with monotone and nonmonotone, nonsmooth material laws and boundary conditions: algorithms and applications. Ph.D. Thesis, Aristotle University, Thessaloniki (1991).

18. G. Duvaut and J. L. Lions, Les Inéquations en Méchanique et en Physique Dunod, Paris (1970) [English translation by Springer, Wien (1972).]

19. I. Hlavaček, J. Haslinger, J. Nečas and J. Loviček, Solution of Variational inequalities in Mechanics. Applied Mathematical Science, Vol. 66. Springer, Wien (1988).

20. P. D. Panagiotopoulos, Hemivariational Inequalities and their Applications in Mechanics and Engineering. Springer, New York (1993).

21. G. E. Stavroulakis and P. D. Panagiotopoulos, Convex multilevel decomposition algorithms for non-monotone problens. Intl $J$. Numer. Meth. Engng 36(11), 1945-1966 (1993).

22. M. Ap. Tzaferopoulos, On an efficient new numerical method for the frictional contact problem of structures with convex energy density. Comput. Struct. 48(1), 87-106 (1993).

23. W. S. Dorn, R. E. Gomory and H. I. Greenberg, Automatic design of optimal structures. J. Mécan. 3(1), 25-52 (1964).

24. M. P. Bendsoe, A. Ben-Tal and J. Zowe, Optimization methods for truss geometry and topology design. Report No. 449. DFG (German Research Society) Programm "Anwendungsbezogene Optimierung und Steuerung", Universität Bayreuth (1993).

25. A. Höfler, U. Leyßner and J. Wiedemann, Optimization of the layout of trusses combining strategies based on Michell's theorem and the biological principles of evolution. In 2nd Symp. on Structural Optimization, AGARD Conf. Proc., Vol. 123, pp. A1-A8 (1973).

26. P. Pedersen, On the minimum mass layout of trusses. In Symp. on Structural Optimization, AGARD Conf. Proc., Vol. 36, pp. 11.1-11.17 (1970).

27. A. G. M. Michell, The limits of economy of material in frame-structures. Phil. Mag. 8(6), 589-597 (1904).

28. M. Fremond, Yield theory in physics. In Topics in Nonsmooth Mechanics (Edited by J. J. Moreau, P. D. Panagiotopoulos and G. Strang), pp. 187-240. Birkhäuser, Boston (1988).

29. J. Mandel, Propriétés Mécaniques des Matériaux. Réologie-Plasticité. Eyrolles, Paris (1978). 
30. R. T. Rockafellar, Convex Analysis. Princeton Press, Princton, NJ (1970).

31. F. H. Clarke, Optimization and Nonsmooth Analysis. Wiley, New York (1983).

32. G. Maier, Limit design in the absence of a given layout: a finite element zero-one programming problem. In Optimum Structural Design. Theory and Applications (Edited by R. H. Gallaher and O. C. Zienkiewicz), pp. 223-239. Wiley, New York (1977).
33. G. Strang, A framework for equilibrium equations. Numerical Analysis Report 87-4, Deptartment of Mathematics, MIT, Cambridge, MA (1987).

34. R. Fletcher, Practical Optimization Methods (2nd edn). Wiley, New York (1987).

35. R. J. Hanson and K. L. Hiebert, A sparse linear programming subprogram. Sandia National Laboratories Report. SAND81-0297 (1981).

36. P. E. Gill, W. Murray and M. H. Wright, Practical Optimization. Academic Press, New York (1981). 